\title{
Redox State Control of Human Cytoglobin by Direct Electrochemical Method to Investigate Its Function in Molecular Basis
}

\author{
Yasuhiro Mie, ${ }^{, a}$ Kyoka Takahashi, ${ }^{a}$ Ryo Torii, ${ }^{b}$ Shen Jingkai, ${ }^{b}$ Takumi Tanaka, ${ }^{b}$ Kenta Sueyoshi, ${ }^{b}$ \\ Hirofumi Tsujino, ${ }^{b}$ and Taku Yamashita ${ }^{c}$ \\ ${ }^{a}$ Bioproduction Research Institute, National Institute of Advanced Industrial Science and Technology (AIST); \\ Sapporo 062-8517, Japan: ${ }^{b}$ Graduate School of Pharmaceutical Sciences, Osaka University; Osaka 565-0871, \\ Japan: and ' School of Pharmacy and Pharmaceutical Sciences, Mukogawa Women's University; Hyogo 663-8179, \\ Japan.
}

Received February 29, 2020; accepted May 14, 2020; advance publication released online May 26, 2020

The direct electron transfer between human cytoglobin (Cygb) and the electrode surface, which would allow manipulating the oxidation states of the heme iron in Cygb, was first observed by immobilizing Cygb on a nanoporous gold (NPG) electrode via a carboxy-terminated alkanethiol. The voltammetric performances of the wild type and mutated Cygb-immobilized NPG electrodes were evaluated in the absence or presence of potential substrates. The obtained results demonstrated that the usefulness of the proposed method in understanding the function of Cygb in molecular basis. electrode

Key words cytoglobin; hypoxic condition; electrochemical assay; nitrite reduction; heme protein; nanoporous

\section{Introduction}

Human cytoglobin (Cygb), which contains a heme as the active site, is the fourth globin protein in mammals and is ubiquitously expressed in many organs. ${ }^{1)}$ Although the true cellular function(s) of Cygb still remains unclear, the upregulation of $\mathrm{Cygb}$ under hypoxic conditions suggests that this molecule protects cells under hypoxic/ischemic conditions. ${ }^{2,3)}$ The activities of Cygb include scavenging of reactive oxygen species, peroxidase activity, lipid oxidation, nitric oxide (NO) oxidation, and $\mathrm{NO}_{2}$ reduction. These activities might be related to the function(s) of Cygb, but their mechanisms are incompletely understood and require in vitro study. ${ }^{4-6)}$ As some of the Cygb activities are expressed via the reduced (ferrous) state of heme iron, they are usually assayed by a reductant such as dithionite. However, this sometimes leads to difficulty in achieving accurate data analysis and precise regulation of the redox states of Cygb.

In recent time, direct electron transfer (DET) reaction between redox proteins and an electrode has been achieved by various strategies. $^{7-14)}$ Electrochemical methods with DET are attractive for applications in biosensors and bioreactors using the redox enzymes. DET methods are also applicable to the molecular-based analyses of protein functions associated with the redox state change of its active site. In the electrochemical method, the oxidized or reduced state of a redox enzyme can be precisely altered by controlling the electrode potential, without adding an oxidizing or reducing agent. Therefore, electrochemical methods are powerful tools for understanding detailed protein functions in the absence and presence of potential ligands, substrates, and other affecting agents. The electron-transfer rate is an exponentially decreasing function

This paper is dedicated to Emeritus Professor Tadayuki Uno at Osaka University, who was dead in 2017 of the distance between the active (redox) site in the protein and the electrode surface, which itself depends on the orientation of the protein; hence DET would be observed only when the protein orientations on the electrode surface are adequately controlled. ${ }^{8)}$ A strategy that inhibits the denaturation of the protein structure on the solid electrode surface is also important. When modified by functional organic layers, the electrode surface can interact with the protein molecules at an orientation that achieves DET. ${ }^{9,10)}$ Recently, many reports have shown that electrodes with various nanostructures, such as nanoparticles, nanorods, nanotubes, nanopores, and nanosheets, can facilitate the DET of redox proteins by minimizing the aforementioned distance..$^{111,12)}$ We also demonstrated that a nanoporous gold (NPG) electrode, which can be facilely produced by a simple anodization method, enables electrochemical control of the redox states of $\mathrm{CYP}^{13)}$ and neuroglobin $\left(\mathrm{Ngb}^{14)}\right.$ the third globin protein) through the DET reaction.

In this study, we applied our NPG electrode system to Cygb, and newly observed an electron transfer reaction between the electrode and the protein by voltammetry. Judging from the clear bioelectrocatalytic responses in the presence of potential substrates, the present system can effectively investigate the molecular basis of Cygb activities coupled with electron transfer, and provide insights into Cygb function(s).

\section{Experimental}

Highly purified Cygb was prepared in an E. coli expression system. ${ }^{15)}$ A mutant Cygb was prepared by site-directed mutagenesis as previously reported. ${ }^{15)}$ The NPG electrode was constructed by anodizing a commercially-available planar gold electrode in a $35-500 \mathrm{mM} \mathrm{HCl}$ solution and the surface area was estimated as $0.7 \mathrm{~cm}^{2}$ (on average) from the reductive charge on the gold surface oxide. ${ }^{13,14)}$ The morphology of the NPG surface was characterized by scanning electron 
microscopy (SEM). Both the planar (non-anodized) gold and the NPG surfaces were modified with 5-carboxy-1-pentanethiol, and the purified Cygb was immobilized on the modified electrode surface through the amino group(s) on the enzyme surface. ${ }^{14)}$ The electrochemical measurements were performed with a configuration consisting of a $\mathrm{Pt}$ wire, an $\mathrm{Ag}|\mathrm{AgCl}|$ sat. $\mathrm{KCl}$, and a Cygb immobilized electrodes, as auxiliary, reference, and working electrodes, respectively, at $25^{\circ} \mathrm{C}$ under Ar atmosphere, using an ALS model 660D electrochemical analyzer. The potentials were converted to potentials versus the standard hydrogen electrode (SHE). The effect of an NO donor, namely, 1-(Hydroxy-NNO-azoxy)-L-proline (PROLI NONOate), on the electrochemical response of Cygb was also investigated.

\section{Results and Discussion}

Redox State Control of Cygb by Direct Electrochemical Method To investigate the DET between Cygb and the electrode, the voltammetry of the Cygb-immobilized NPG electrode was compared with that of a Cygb-immobilized planar gold electrode. The representative surface morphology of the NPG electrode was shown together with that of planar gold electrode (Figs. 1A, B). The NPG was composed of ligaments and pores as described in previous reports, ${ }^{14,16)}$ and the ligament sizes were approx. $50 \mathrm{~nm}$. The obtained voltammograms of the Cygb-immobilized electrodes were shown in Figs. 1C and D. At the Cygb-immobilized planar gold electrode, no clear faradaic signal appeared (Fig. 1C). On the other hand, Cygb-immobilized NPG electrode exhibited a well-defined couple of current peaks with a formal potential, $E^{0 \prime}$, of $-0.035 \mathrm{~V}$ at $\mathrm{pH} 6.0$ (Fig. 1D). The surface density of electrochemically active Cygb molecules on the NPG electrode surface was $1.8 \mathrm{pmol} \mathrm{cm} \mathrm{cm}^{-2}$, consistent with a monolayer coverage. The obtained $E^{0 \prime}$ value was close to that $(-0.037 \mathrm{~V})$ afforded when a freely diffusing Cygb was subjected to a titration method at $\left.\mathrm{pH} 7.0,{ }^{17}\right)$ demonstrating that the present method can evaluate the redox chemistry of Cygb. The considerably enhanced electrochemical signals of Cygb on the NPG electrode compared with a planar gold electrode could arise from the curvature effect ${ }^{18)}$ of the nanoporous structure. A curved surface would admit more Cygb molecules with orientations that favor electron transfer with the electrode surface; moreover, the enlarged electrode surface area of the NPG would increase the total amount of immobilized Cygb.

Electron Transfer Kinetics of Cygb Using the Cygbimmobilized NPG electrode, the potential scan rate dependences of the voltammetric peak currents and potentials were analyzed (Fig. 2). The peak currents in both the cathodic and anodic directions linearly increased with the scan rate (Fig. 2A), indicating the electrochemical responses from the surface-confined Cygb. The separation between the cathodic and anodic peak potentials increased as the scan rate increases as shown in Fig. 2B (trumpet plot). From this plot, the apparent heterogeneous electron transfer rate constant $\left(k_{\mathrm{s}}{ }^{\prime}\right)$ was estimated to be $1 \mathrm{~s}^{-1}$ using a simulation program. ${ }^{19)}$ This value was significantly smaller than that $\left(46 \mathrm{~s}^{-1}\right)$ obtained for human Ngb-immobilized NPG electrode prepared by the same immobilization strategy. ${ }^{14)}$ Although both globins have a six-coordinated heme iron and catalyze similar reactions as mentioned above, ${ }^{1-6)}$ the slower electron transfer kinetics of Cygb implies that $\mathrm{Cygb}$ inherently has a larger structural reorganization
(A)

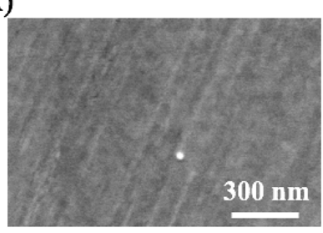

(C)

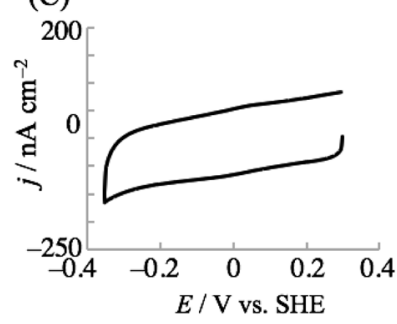

(B)
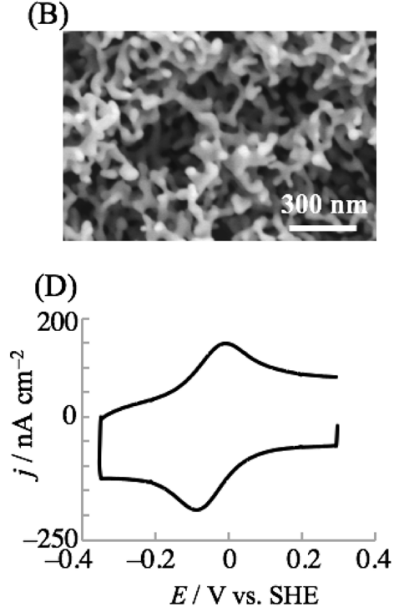

Fig. 1. (A, B) Representative Surface Morphologies of the Planar Gold (A) and NPG (B) Electrodes Observed by SEM; (C, D) Cyclic Voltammograms Obtained at Cygb-Immobilized Planar Gold (C) and NPG (D) Electrodes in 0.1 M Bis-Tris Buffer Solution ( $\mathrm{pH}$ 6.0) Containing $100 \mathrm{mM}$ $\mathrm{NaCl}$

Cygb was attached on each surface via the amino groups of the protein molecules. Potential scan rate: $0.05 \mathrm{~V} \mathrm{~s}^{-1}$.
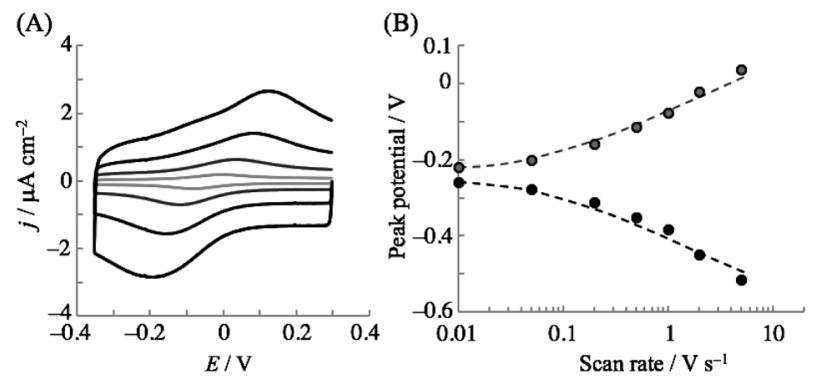

Fig. 2. (A) Cyclic Voltammograms of a Cygb-Immobilized NPG Electrode Obtained at Different Potential Scan Rates in 0.1 M Bis-Tris Buffer Solution ( $\mathrm{pH}$ 6.0) Containing $100 \mathrm{mM} \mathrm{NaCl}$

Scan rates $\left(\mathrm{V} \mathrm{s}^{-1}\right)$ : 0.05 (thin gray line), 0.2 (gray line), 0.5 (dark gray line), 1 (black line); (B) Relationship between peak potentials and scan rates derived from the Cygb-Immobilized NPG electrodes. Black and gray circles indicate the cathodic and anodic peak potentials in the voltammograms, respectively.

around the active site compared to that of $\mathrm{Ngb}$. The result agrees with the fact that Cygb has a slower autoxidation rate $\left(7.5 \times 10^{-2} \mathrm{~min}^{-1}\right.$ in the fast phase and $7.2 \times 10^{-3} \mathrm{~min}^{-1}$ in the slow phase at $\mathrm{pH} 7.4$ and $25^{\circ} \mathrm{C}$ ) than that (approx. $0.17 \mathrm{~min}^{-1}$ ) of Ngb. ${ }^{20-22)}$ These kinetic differences between the two globins may differentiate the function(s) of the globins.

Electrochemical Assay of Wild Type (WT) and Mutated Cygbs As described, DET reaction between Cygb and the electrode has been demonstrated, then we examined the electrocatalytic reactions of Cygb on two potential substrates $\left(\mathrm{H}_{2} \mathrm{O}_{2}\right.$ and nitrite). Recently, the intramolecular disulfide bond between cysteines, Cys38 and Cyc83, has been shown to affect the affinity for exogenous ligands and the catalytic activity of Cygb. ${ }^{21,22)}$ To understand the effect of disulfide formation/ breakage on the electrochemical properties of Cygb, the C38S and C83S double mutant (2CS) was also studied in addition to WT. Figures $3 \mathrm{~A}$ and B show the voltammograms of the WT and mutant electrode preparations, respectively, in the presence and absence of $\mathrm{H}_{2} \mathrm{O}_{2}$. The $2 \mathrm{CS}$ mutant exhibited a clear redox-peak couple as in the case of WT in the absence of $\mathrm{H}_{2} \mathrm{O}_{2}$. The redox potential of the mutant was estimated 
as $-0.040 \mathrm{~V}$ (Fig. 3B), which was very close to that of WT. Therefore, the intramolecular disulfide bridge exerted little effect on the redox potential. This suggests that the corresponding disulfide formation/breakage does not significantly alter the strength of the axially coordinated ligands to the heme iron. One of these ligands, His81, is a distal one that approaches the Cys 83 residue. When an exogenous ligand binds to the heme iron in Cygb, the His81 ligand must dissociate from the iron; therefore, the strength of the His coordination could be an important determiner for the ligand binding property. Astudillo et al. reported ${ }^{23)}$ that the disulfide bridge modulates the conformational dynamics in Cygb including the size and energy barrier between the internal hydrophobic sites. Hence, the present electrochemical result indicates that the difference in exogenous ligand affinity with and without the intramolecular disulfide bridge would not result from the perturbation of His 81 coordination but from the structural changes around the distal pocket altering the ligand migration pathway. ${ }^{4,23)}$

Upon addition of $\mathrm{H}_{2} \mathrm{O}_{2}$, the electrocatalytic current in the cathodic direction at the redox potential of the heme iron in Cygb was clearly observed in a $\mathrm{H}_{2} \mathrm{O}_{2}$ concentration-dependent manner for both WT and 2CS mutant (Figs. 3A, B). When the $\mathrm{H}_{2} \mathrm{O}_{2}$ was added, no faradaic current was detected at the carboxyalkanethiol-modified NPG electrode within the potential region in Fig. 3. ${ }^{14)}$ The $\mathrm{H}_{2} \mathrm{O}_{2}$ catalytic reaction has been widely recognized in other hemoproteins such as hemoglobin, myoglobin, and neuroglobin, where the electrochemically reduced heme iron was re-oxidized chemically by the $\mathrm{H}_{2} \mathrm{O}_{2}$ to produce the catalytic current ${ }^{14,24,25)}$; hence, it was reasonably observed for both Cygb WT and 2CS regardless of the intramolecular disulfide bond. Thus, the electrocatalytic reaction of Cygb on its capable substrate was clearly demonstrated using the NPG electrode.

Cygb is known to show nitrite reductase activity under hypoxic conditions, ${ }^{22,26)}$ which is expected to generate NO to facilitate vasodilation and increase blood flow to the hypoxic tissue. Panels C and D of Fig. 3 show the voltammograms of WT and 2CS, respectively, upon the addition of nitrite under the $\mathrm{Ar}$ atmosphere at $0.1 \mathrm{~V} \mathrm{~s}^{-1}$. For WT, a significant increase in the cathodic current was clearly detected at the redox potential of Cygb. As no significant voltammetric signal was detected at the carboxyalkanethiol-modified NPG electrode, the nitrite must have been reduced by the electrochemically reduced ferrous Cygb. On the other hand, the 2CS mutant did not exhibit such an increase in the current. These electrochemical results coincide with the previous report that Cygb without intramolecular disulfide bond showed much slower nitrite reductase activity (50 times smaller rate constant) ${ }^{22)}$ than that of Cygb with the disulfide bridge, which was revealed with spectroscopic method. Note that the electrocatalytic current generated by the Cygb-immobilized NPG electrode in nitrite concentration-dependent manner was only observed at the concentration lower than around $500 \mu \mathrm{M}$. Increasing the nitrite concentration beyond this level diminished the catalytic current, as evidenced by the smaller signals than those of the initial state (Fig. 3C, gray dotted line). Although this observation is poorly understood at present, previous work suggested that NO molecules stably bind to ferrous Ngb and the adduct exists in a less active (frozen) state under hypoxic conditions. ${ }^{27)}$ To investigate whether NO molecules affect the elec-
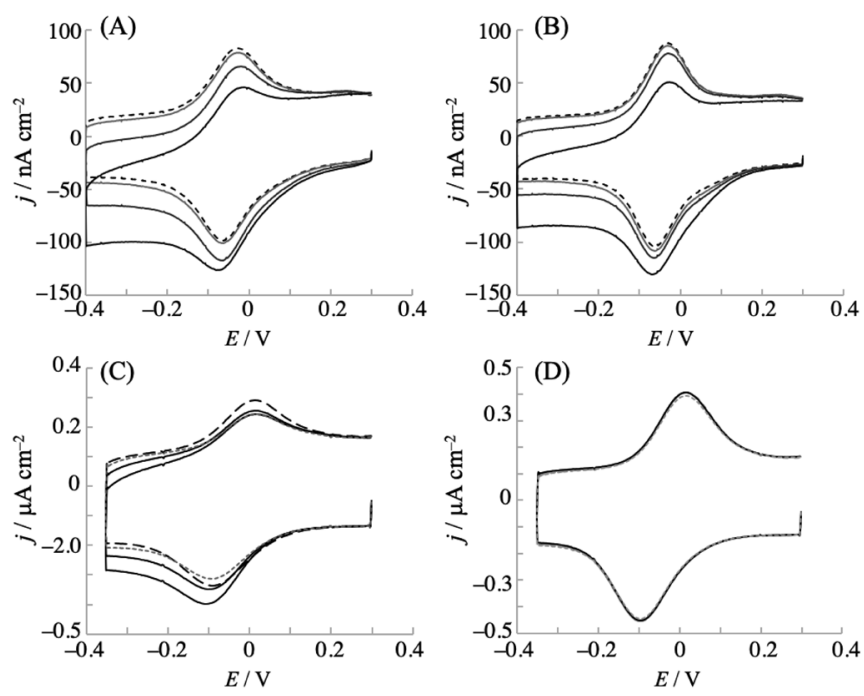

Fig. 3. Cyclic Voltammograms Obtained at Cygb-Immobilized NPG Electrodes for WT $(A, C)$ and $2 \mathrm{CS}$ Mutant $(\mathrm{B}, \mathrm{D})$ upon Addition of $\mathrm{H}_{2} \mathrm{O}_{2}$ (A, B), and Nitrite (C, D) Obtained in $0.1 \mathrm{M}$ Bis-Tris Buffer $(\mathrm{pH} 6.0)$

Dashed black lines were without $\mathrm{H}_{2} \mathrm{O}_{2}$ or nitrite. $\left[\mathrm{H}_{2} \mathrm{O}_{2}\right.$ ]: $0,10,30 \mu \mathrm{M}$. [nitrite] $0,100,500$, and $2000 \mu \mathrm{M}$. Dotted gray curve was obtained in $2000 \mu \mathrm{M}$ of nitrite. Scan rates in panels $(\mathrm{A}, \mathrm{B})$ and $(\mathrm{C}, \mathrm{D})$ were 0.02 and $0.1 \mathrm{~V} \mathrm{~s}^{-1}$, respectively.
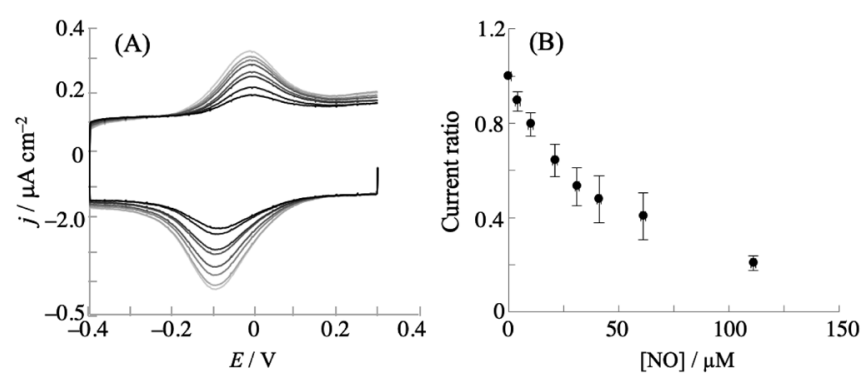

Fig. 4. (A) Cyclic Voltammograms of the Cygb-Immobilized NPG Electrodes before and upon Addition of NO Donor (Light to Dark Lines Indicate Increasing NO Concentration) Obtained in $0.1 \mathrm{M}$ Bis-Tris Buffer (pH 6.0) at $0.1 \mathrm{~V} \mathrm{~s}^{-1}$; (B) Observed Current Ratio (Normalized by the Ratio in the Absence of NO) versus NO Concentration, Derived from the Voltammograms

The current ratio is represented as the mean \pm standard deviation $(n=3$ independent experiments).

trochemical response of Cygb, we conducted the voltammetry of the Cygb-immobilized NPG electrode in the presence of an NO donor. As shown in Figs. 4A and B, the faradaic current signals in the voltammogram clearly decreased with increasing NO concentration, indicating that NO inhibits the electron transfer reaction of Cygb. Hence, we speculate that the NO molecules produced by the nitrite reduction of $\mathrm{Cygb}$ inhibit its catalytic reaction, which could imply that the NO generation by Cygb in the cytoplasm is tuned by this inhibition mechanism.

\section{Conclusion}

We manipulated the redox state of human Cygb by a direct electrochemical method using an NPG electrode. The obtained electrochemical characteristics provide novel information for understanding Cygb functionality. As the NPG electrode can be facilely prepared by a simple strategy, the proposed system is expected as a useful tool for investigating the molecular basis of Cygb function(s). 
Acknowledgments This work was supported in part by the JSPS KAKENHI Grant Numbers JP16K08218 and JP19K07024.

Conflict of Interest The authors declare no conflict of interest.

\section{References}

1) Nakatani K., Okuyama H., Shimahara Y., Saeki S., Kim D. H., Nakajima Y., Seki S., Kawada N., Yoshizato K., Lab. Invest., 84, 91-101 (2004).

2) Fordel E., Thijs L., Martinet W., Lenjou M., Laufs T., Van Bockstaele D., Moens L., Dewilde S., Neurosci. Lett., 410, 146-151 (2006).

3) Emara M., Turner A. R., Allalunis-Turner J., Cancer Cell Int., 10, 33 (2010).

4) Reeder B. J., Svistunenko D. A., Wilson M. T., Biochem. J., 434, 483-492 (2011)

5) Beckerson P., Wilson M. T., Svistunenko D. A., Reeder B. J., Biochem. J., 465, 127-137 (2015)

6) Yamashita T., Hafsi L., Masuda E., Tsujino H., Uno T., Chem. Pharm. Bull., 62, 613-615 (2014).

7) Pinyou P., Blay V., Muresan L. M., Noguer T., Mater. Horizons, 6, 1336-1358 (2019)

8) Kaida Y., Hibino Y., Kitazumi Y., Shirai O., Kano K., Electrochem. Commun., 98, 101-105 (2019).

9) Taniguchi I., Yoshimoto S., Nishiyama K., Chem. Lett., 26, 353-354 (1997).

10) Holland J. T., Lau C., Brozik S., Atanassov P., Banta S., J. Am. Chem. Soc., 133, 19262-19265 (2011).

11) Takahashi Y., Kitazumi Y., Shirai O., Kano K., J. Electroanal.
Chem., 832, 158-164 (2019).

12) Suzuki M., Murata K., Nakamura N., Ohno H., Electrochemistry, 80, 337-339 (2012)

13) Mie Y., Ikegami M., Komatsu Y., Chem. Lett., 45, 640-642 (2016).

14) Mie Y., Takahashi K., Itoga Y., Sueyoshi K., Tsujino H., Yamashita T., Uno T., Electrochem. Commun., 110, E106621 (2020).

15) Tsujino H., Yamashita T., Nose A., Kukino K., Sawai H., Shiro Y., Uno T., J. Inorg. Biochem., 135, 20-27 (2014).

16) Mie Y., Yasutake Y., Ikegami M., Tamura T., Sens. Actuators $B$ Chem., 288, 512-518 (2019).

17) Corti P., Ieraci M., Tejero J., Nitric Oxide-Biol. Chem., 53, 22-34 (2016).

18) Sugimoto Y., Takeuchi R., Kitazumi Y., Shirai O., Kano K., J. Phys. Chem. C, 120, 26270-26277 (2016).

19) Armstrong F. A., Camba R., Heering H. A., Hirst J., Jeuken L. J. C., Jones A. K., Leger C., McEvoy J. P., Faraday Discuss., 116, 191-203, discussion, 257-268 (2000).

20) Fago A., Hundahl C., Dewilde S., Gilany K., Moens L., Weber R. E., J. Biol. Chem., 279, 44417-44426 (2004).

21) Hanai S., Tsujino H., Yamashita T., Torii R., Sawai H., Shiro Y., Oohora K., Hayashi T., Uno T., J. Inorg. Biochem., 179, 1-9 (2018).

22) Reeder B. J., Ukeri J., Nitric Oxide-Biol. Chem., 72, 16-23 (2018).

23) Astudillo L., Bernad S., Derrien V., Sebban P., Miksovska J., J. Inorg. Biochem., 129, 23-29 (2013).

24) Yang W. W., Li Y. C., Bai Y., Sun C. Q., Sens. Actuators B Chem., 115, 42-48 (2006).

25) Shang L., Sun Z., Wang X., Li G., Anal. Sci., 19, 1537-1539 (2003).

26) Li H. T., Hemann C., Abdelghany T. M., El-Mahdy M. A., Zweier J. L., J. Biol. Chem., 287, 36623-36633 (2012).

27) Brunori M., Giuffre A., Nienhaus K., Nienhaus G. U., Scandurra F. M., Vallone B., Proc. Natl. Acad. Sci. U.S.A., 102, 8483-8488 (2005). 